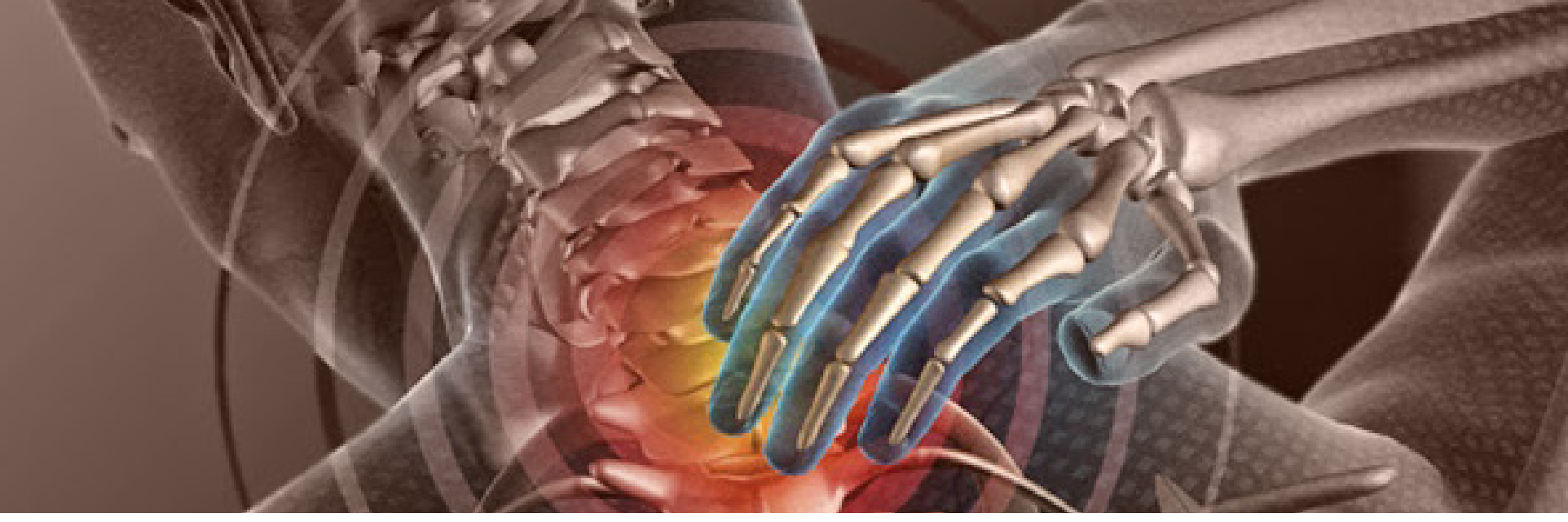

\title{
MOLESTIAS MUSCULO ESQUELÉTICAS Y EVALUACIÓN DE POSTURAS DE TRABAJO EN ENFERMERAS DE CIRUGÍA: ESTUDIO PILOTO
}

MUSCULOSKELETAL DISCOMFORT AND EVALUATION OF NURSE WORKING POSTURE IN SURGERY: A PILOT STUDY

\author{
MARÍA OLGA QUINTANA ZAVALA.*
}

\section{ABSTRACT}

Objective: To identify ergonomic risk factors in nurses. Method: observational, descriptive, crosssectional pilot study. The sample was composed of 4 nurses. Borg scale was used to measure the intensity of the trouble and method of Ovako Working Posture Analysing System for classifying positions. Results: The total number of participants present discomfort in any part of the body with mild to moderate. Conclusions: the discomfort perceived by nurses could be related to the positions taken during the performance of work activities, as it is documented that the pain in the back of the neck, pain in the posterior region of the shoulders, pain in the lumbar region and pain in the popliteal region and calves are associated with recurring injuries in nursing work.

Keywords: Evaluation of posture, skeletal muscle discomfort

\section{RESUMEN}

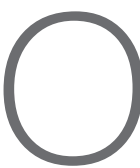
bjetivo: identificar factores de riesgo ergonómicos en enfermeras. Método: estudio piloto observacional, descriptivo y transversal. La muestra se conformó por 4 enfermeras. Se utilizó la escala de Borg para medir la intensidad de la molestia y el método de Ovako Working Posture Analysing System para clasificar las posturas. Resultados: el total de las participantes presentan molestia en alguna región del cuerpo con intensidad de leve a moderada. Conclusiones: las molestias percibidas por enfermeras podrían estar relacionadas a las posturas que adoptan durante la realización de actividades laborales, ya que está documentado que el dolor en la región posterior del cuello, dolor en la región posterior de los hombros, dolor en la región lumbar y dolor en la región poplítea y pantorrillas, se asocian a lesiones recurrentes en el trabajo de enfermería.

Palabras Clave: Evaluación de postura, Molestias musculo esqueléticas

${ }^{*}$ ) Doctora en enfermería. Docente de tiempo completo. Departamento de Enfermería. Universidad de Sonora. Email: olga_quintana@enfermeria.uson.mx 


\section{INTRODUCCIÓN}

Se entiende como factor de riesgo a cualquier característica presente en el ambiente laboral, que al actuar sobre el trabajador puede producir una respuesta, en la forma de incomodidad, dolor o lesión (1). El trabajo que realizan los profesionales de enfermería dentro de los hospitales es el que incorpora más factores de riesgo a la salud, comparado con otros grupos de profesionales sanitarios, entre ellos se encuentran los riesgos: psicosociales, biológicos, físico-químicos, de seguridad y ergonómicos ${ }^{(2,3)}$.

En este sentido el desarrollo de una solución que resulte en mejores condiciones de trabajo para las enfermeras, debe incluir el análisis del sistema y su contexto, así como del individuo y su situación ${ }^{(4)}$.

La ergonomía es una multidisciplina preocupada de la adaptación del trabajo al ser humano, que tiene como objetivos promover la salud y el bienestar, reducir los accidentes y mejorar la productividad ${ }^{(5)}$. Y se divide en cinco áreas: ambiental, geométrica, temporal, de comunicación, seguridad, de concepción, de corrección y de protección ${ }^{(6)}$.

Existen algunos riesgos ergonómicos en el ejercicio de la enfermería: a) la carga física o sobre esfuerzo físico y/o postural, b) los requerimientos excesivos de fuerza, que superan la capacidad de la enfermera o enfermero, c) los requerimientos excesivos de movimiento, que se dan cuando el movimiento se realiza sobre una carga estática alta y d) las condiciones inadecuadas de los puestos de trabajo, lo que ocasiona recorridos innecesarios, accidentes de trabajo y errores ${ }^{(2)}$.

El equipo de enfermería enfrenta condiciones laborales inadecuadas en su medio ambiente de trabajo, que pueden dar lugar a riesgos ergonómicos: la organización del trabajo (jornada excesiva, déficit de profesionales), los factores relacionados con el medio ambiente (mobiliario y equipos inadecuados y obsoletos), y sobrecargas en los segmentos corporales. Estos riesgos generan gran problema de salud pública debido a su relación con el absentismo, los permisos y el retiro por discapacidad ${ }^{(7)}$.

Estudios realizados al respecto de los riesgos ergonómicos en enfermería muestran que sufren lesiones musculo-esqueléticas que pueden estar relacionadas con la insuficiencia de las condiciones ambientales de trabajo ${ }^{(8)}$, permanecer de pie durante las cirugías, ${ }^{(9)}$ se ha identificado prevalencia de dolor de espalda en enfermeras, ${ }^{(10)}$ los autores recomiendan utilizar equipo ergonómico en los cuidados de enfermería ${ }^{(11)}$.

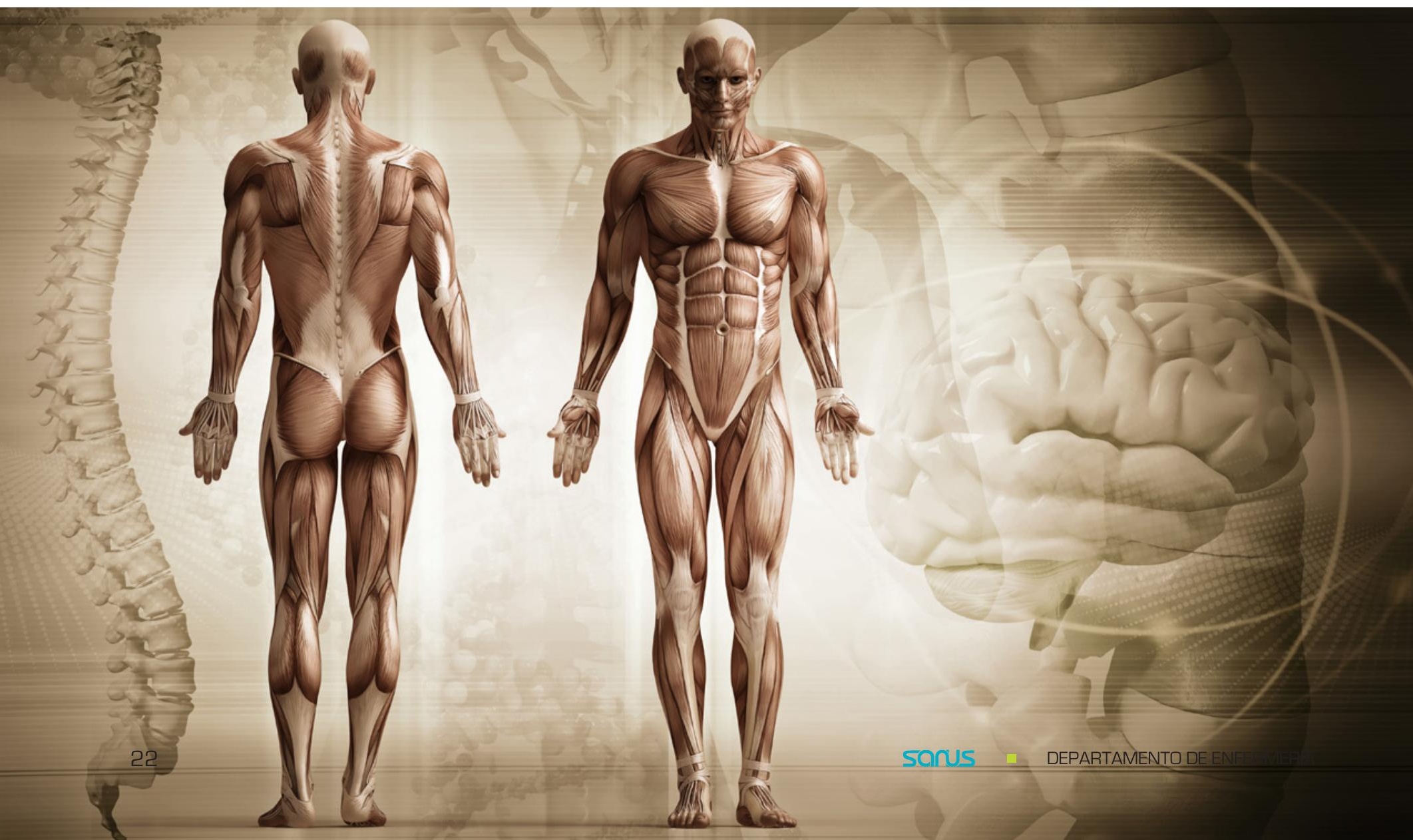


En cuanto a turno de trabajo $75 \%$ labora mañana y tarde y $25 \%$ labora en cuarto turno (largo, noche, libre-libre), coincidente con el estado civil donde se observó que $75 \%$ es soltera y $25 \%$ casada, ninguna tiene hijos.

Tocante a las molestias musculo esqueléticas que presentan indagadas por medio del esquema elaborado por Corllet y Bishop ${ }^{(13)}$, se identificó que el total de las participantes presentan molestia en alguna región del cuerpo, entre ellas la región posterior del cuello, coincidente con lo reportado por investigadores en Venezuela quienes afirman que las más altas prevalencias de molestias identificadas en las enfermeras de un hospital militar se relacionan al dolor de cuello, lo que se asocia a trabajar a una altura inadecuada $(17,18)$.

Así también las enfermeras refieren dolor en la región posterior de los hombros, igual que lo reportado por autores Colombianos, donde lo incluyen como una de las lesiones musculo esqueléticas más frecuentes en trabajadores ${ }^{(19)}$.

Del mismo modo, el dolor en la región lumbar, fue referido por las enfermeras participantes, coincidente con lo reportado por Morata y Pérez ${ }^{(20)}$, quienes afirman que el dolor lumbar es el más frecuente expresado por el personal sanitario que cuida a los pacientes, así como Peña y Solano ${ }^{(21)}$ quienes mencionan que las enfermeras profesionales presentan altos índices de dolor de espalda de origen ocupacional.

Se observó además que una de las participantes presenta dolor en la región poplítea y pantorrillas, lo que se puede relacionar con los resultados de otros estudios que afirman que algunas enfermeras permanecen en una postura fija por tiempo prolongado, sin realizar pausa o descanso (22).

La intensidad de las molestias fue medida con la escala de intensidad de la molestia de Borg ${ }^{(14)}$, resultando con intensidad de leve a moderada, dentro del último año y fue atribuido por las propias participantes a tensión, posturas y problemas de circulación venosa.

Para la evaluación de las posturas adoptadas durante el trabajo de enfermeras en el área de cirugía, se utilizó el método OWAS el cual se basa en una clasificación simple y sistemática de las posturas de trabajo, combinando observaciones sobre las tareas, con el objetivo de realizar una evaluación del riesgo de carga postural en términos de frecuencia y gravedad.

El análisis de los datos obtenidos por las observaciones a enfermeras durante la realización de las tareas se efectuó después de la introducción de los datos en un software de ergonomía el cual calcula el tipo de riesgo ${ }^{(13)}$.

\section{Postura adoptada por enfermeras durante la curación de herida quirúr- gica a usuarios postrados en cama.}

Se observó a cuatro enfermeras mientras realizaban curación de herida quirúrgica a usuarios postrados y a través del método OWAS, se efectuó el análisis de riesgo postural, encontrando espalda inclinada hacia adelante, los dos brazos por debajo del hombro, de pie con las dos piernas derechas y con carga menor de $10 \mathrm{~kg}$. (Fotografías 1 y 2 ).
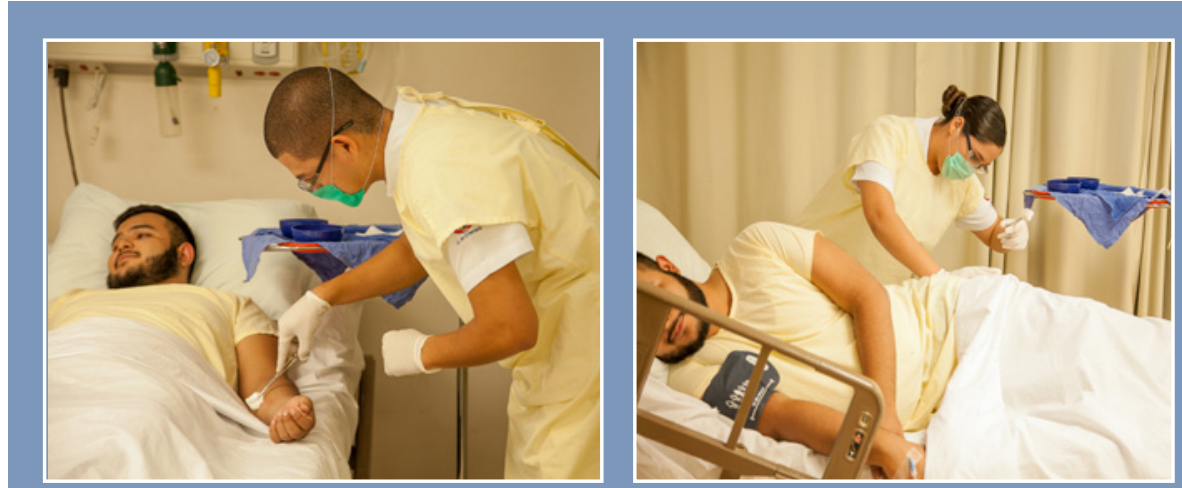

Fotografías 1 Y 2. POSTURA ADOPTADA POR ENFERMERAS AL REALIZAR CURACIÓN DE HERIDA QUIRÚRGICA A USUARIOS POSTRADOS.

El resultado obtenido fue de riesgo nivel 2 en todas las observaciones de posturas adoptadas para la curación de heridas quirúrgicas, lo que significa que la postura representa una posibilidad de causar daño al sistema músculo-esquelético y requiere acciones correctivas en un futuro cercano. Dentro de las condiciones del área física, material y equipo de trabajo se encontró como inadecuada que la cama no permite dar posición apropiada para efectuar el procedimiento.

\section{Postura adoptada por enfermeras para el manejo de bomba de infusión continúa.}

Se observó a cuatro enfermeras mientras realizaban el manejo de bombas de infusión continuas a través del método OWAS, se efectuó el análisis de riesgo postural, encontrando en tres de ellas, espalda derecha, los dos brazos por debajo del hombro, de pie con las dos piernas derechas y con carga menor de $10 \mathrm{~kg}$. (Fotografías 3 y 4).

El resultado obtenido fue de riesgo nivel 1 en todas las observaciones de posturas adoptadas para el manejo de bombas de infusión continua, lo que significa que la postura no representa una posibilidad de causar daño al sis- 


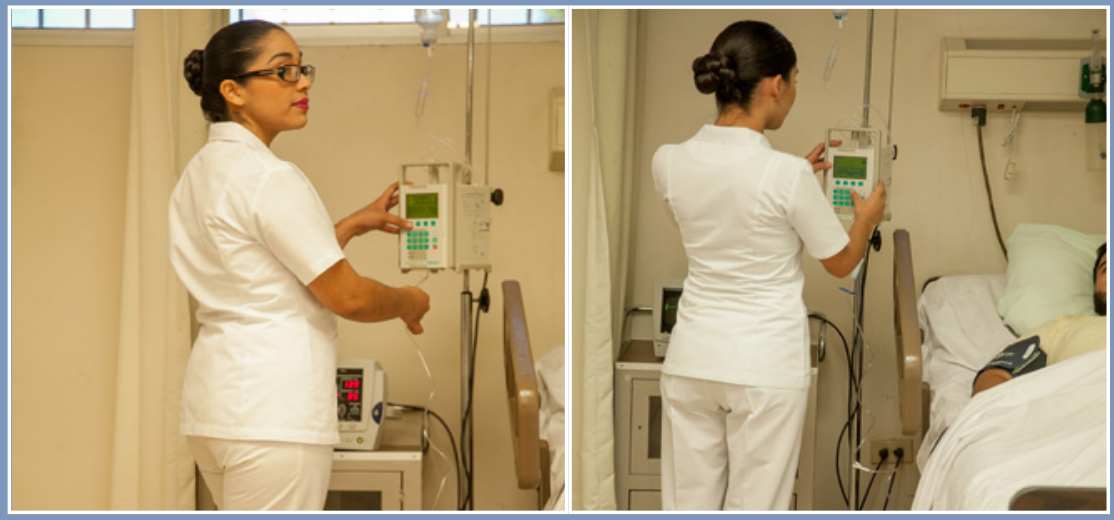

Fotografías 3 Y 4. Postura Adoptada para REALIZAR EL MANEJO DE BOMBA DE INFUSIÓN CONTÍNUA

tema músculo-esquelético y no requiere acciones correctivas. Sin embargo el lugar de colocación de la bomba de infusión continua es muy alto y está situado a la cabecera de la cama de los usuarios, lo cual obliga a la enfermera a mantener los dos brazos por encima de los hombros para su manejo y además implica riesgo para el usuario.

\section{Postura adoptada por enfermeras para la realización de actividades ad- ministrativas.}

Se observó a cuatro enfermeras mientras realizaban labores administrativas a través del método OWAS, se efectuó el análisis de riesgo postural, encontrando que para realizar actividades administrativas las enfermeras adoptaban múltiples posturas, por lo que a continuación se describen.

\section{Inventario de material.}

Espalda recta hacia delante, los dos brazos por debajo del hombro, las piernas arrodilladas y sin carga. El resultado obtenido en cuanto a la evaluación de riesgo postural, fue de riesgo nivel 2 , lo que significa que la postura representa una posibilidad de causar daño al sistema músculo-esquelético y requiere acciones correctivas en un futuro cercano. Fotografía 5.

Labores de gestión asistencial. Espalda de lado, brazos los dos por debajo de los hombros, elevación de un hombro, sentada con solo un pie apoyado sobre el suelo. Riesgo 2. Deben tomarse medidas correctivas en corto tiempo para mejorar las posturas de trabajo Fotografía 6.
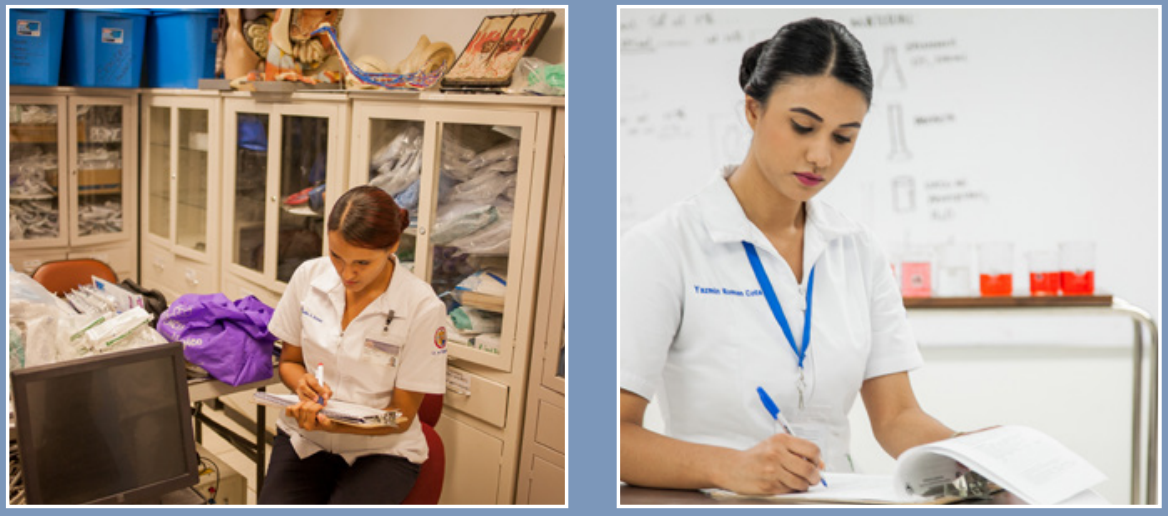

Fotografías 5 Y 6. ENFERMERA REALIZANDO EL INVENTARIO DE MATERIAL 


\section{Pedido de insumos.}

Espalda derecha, brazos los dos por debajo del hombro, de pie con las dos piernas derechas, con carga menor de $10 \mathrm{~kg}$. Riesgo tipo 1 . No es necesaria intervención. Fotografía 7.

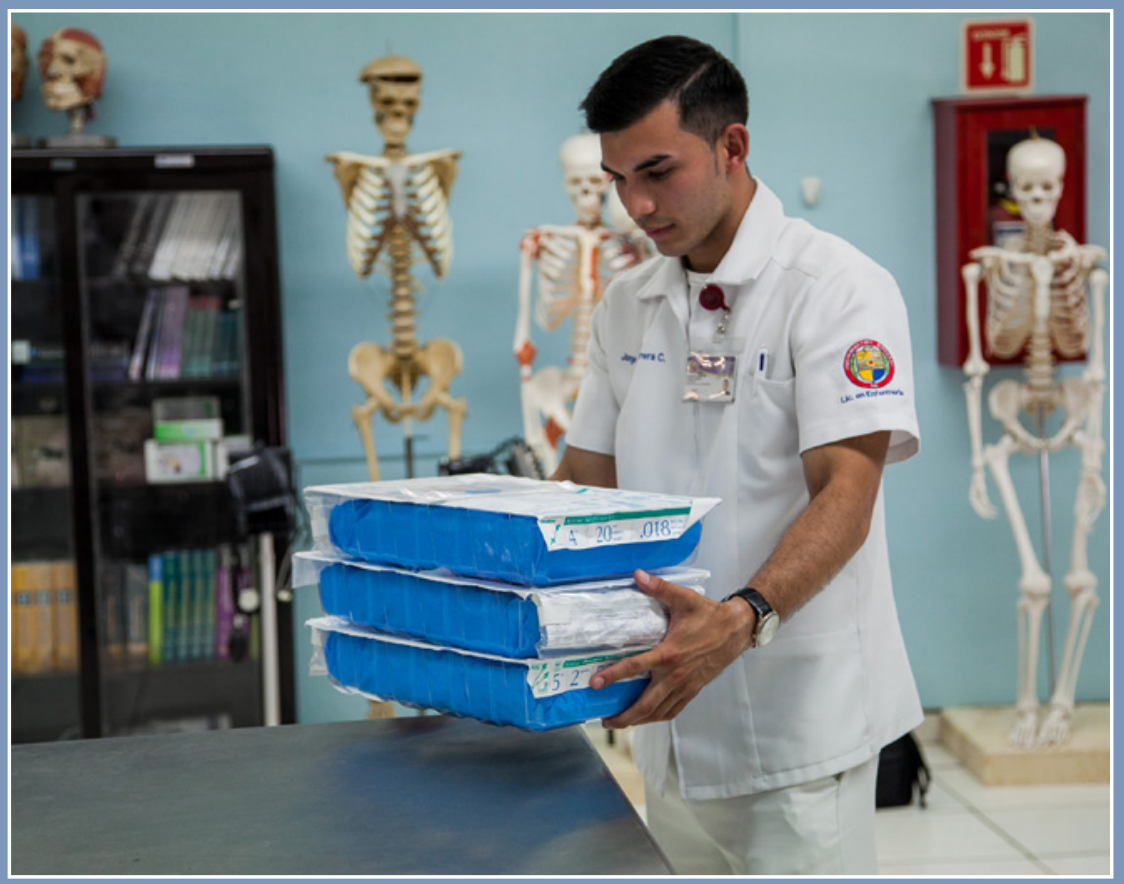

Fotografía 7. Pedido de insumos.

\section{CONCLUSIONES}

La percepción de molestias musculo-esqueléticas de profesionales de enfermería que laboran en el servicio de cirugía, consiste en molestias de intensidad leve a moderada, que podrían estar relacionadas a las posturas que adoptan durante la realización de actividades laborales, ya que está documentado que el dolor en la región posterior del cuello, dolor en la región posterior de los hombros, dolor en la región lumbar y dolor en la región poplítea y pantorrillas, se asocian a lesiones recurrentes en el trabajo de enfermería, sin embargo las participantes son profesionales con poca antigüedad laboral, razón que podría justificar que la intensidad del dolor leve a moderada, es posible que al aumentar la antigüedad, si las posturas no son corregidas la intensidad de las molestias se incremente.

De las tres posturas evaluadas, dos de ellas evidenciaron riesgos tipo 2 (curación de heridas y las labores administrativas: gestión asistencial y realización de inventario de material), por lo que la carga postural puede tener efecto perjudicial en el sistema musculo esquelético y se requiere tomar medidas correctivas para mejorar las posturas de trabajo.

El manejo de bomba de infusión continua presento riesgo tipo 1 , lo que significa que esta postura no necesita ser corregida, sin embargo, una enfermera refiere omalgia, lo que se puede atribuir a la elevación de los dos brazos por arriba del hombro. 


\section{RECOMENDACIONES}

\section{Dirigidas a las enfermeras:}

- Realizar evaluaciones de las posturas de manera sistemática y orientación personalizada sobre mecanica corporal

- Incluir el tema de sobrecarga postural en sesiones clínicas

Mejora de mobiliario:

- Cambiar las camas de los pacientes por camas que permitan cambios de posición, para que al momento de hacer las curaciones la enfermera pueda mantener la espalda recta.
- Instalar los estantes para materiales a una altura tal que la enfermera no tenga que colocarse en cuclillas para realizar el inventario.

- Utilizar un tipode para colocar las bombas de infusion para que la enfermera no tenga que elevar los brazos por encima de los hombros para su manejo.

- Cambiar las sillas de las enfermeras por las que incluyan respaldo, ajustables en altura del asiento, ademas colocar descansa pies, al puesto de trabajo.

\section{REFERENCIAS}

1. Ministerio del Trabajo y Previsión Social. Gobierno de Chile. 2008. Guía Técnica para la evaluación y control de los riesgos asociados al manejo o manipulación manual de carga.

2. Mateu N, Patricio $B$, Farré $C$, Mas $M$. Protegiéndonos ganamos en salud y ahorramos en recursos. Paraninfo digital. 2011; 12.

3. Castillo J. Ergonomía fundamentos para el desarrollo de soluciones ergonómicas. Colección textos. Escuela de Medicina y Ciencias de la Salud. Editorial Universidad. 2010; Colombia. 216 p

4. Montoya M, Palucci M, Cruz M, Taubert F. Lesiones osteomusculares en trabajadores de un hospital mexicano y la ocurrencia del ausentismo. CIENCIA Y ENFERMERIA. 2010; 16 (2):35-46.

5. García L. Observación ergonómica de la jornada de trabajo del profesional de enfermería. Reduca (Enfermería, Fisioterapia y Podología) Serie trabajos Fin de Master. 2009; 1(2): 99-112.

6. De Souza C, Lima da Silva J, Antunes E, Schumacher K, De Almeida T. Riesgos ergonómicos por esfuerzo repetitivo del personal de enfermería en el hospital. Enfermería Global. 2011; No. 23; 251-6.

7. Montoya M, Palucci M, Cruz M, Taubert F. Lesiones osteomusculares en trabajadores de un hospital mexicano y la ocurrencia del ausentismo. CIENCIA Y ENFERMERIA. 2010; 16 (2):35-46.

8. Hughes N, Nelson A, Matz M, Lloyd J. Safe Patient Handling and Movement Series. AORN Ergonomic Tool 4: Solutions for Prolonged Standing in Perioperative Settings. AORN Journal. 2011; 93(6):767-74.

9. Sikiru L, Shmaila H. Prevalence and risk factors of low back pain among nurses in Africa: Nigerian and Ethiopian specialized hospitals survey study. East African Journal of Pablic Health. 2009; 6 (1): 22-25

10. Stricevic J, Balantic Z, Turk Z, Celan D. Ergonomic Analysis of Workload

Diminution by the Use of Assistive Technical Equipment at Nursing Care. HealthMED. 2009; 3 (3)

11. El método OWAS para la evaluación de posturas de trabajo.[internet].[Recuperado en Noviembre del 2011]. Disponible en: www.fi.uba. ar/archivos/posgrados_apuntes_Metodo_OWAS
12. Ergonatuas.com. Departamento de Proyectos de Ingeniería de la Universidad Politécnica de Valencia. [Internet].[Recuperado en Noviembre del 2011] Disponible en: http://www.ergonautas.upv.es/controlusuarios/registrarse.php

13. Corlett E. N, Bishop R. P. A technique for assessing postural discomfort. Ergonomics. 1976; 19(2), 175-82.

14. Borg G. Borg's Perceived exertion and pain scales. Library of congress cataloging in publication data. 1998; 1-113.

15. Emanuel E. ¿Qué hace que la investigación clínica sea realmente ética? siete requisitos éticos. Pautas Éticas de Investigación en Sujetos Humanos: Nuevas Perspectivas. [Internet]. [Consultado Octubre 2011]. Disponible en: http://cursos.puc.cl/enf1123-1/almacen/1306257874_mcatoni_sec1_pos0.pdf

16. Biblioteca del Congreso Nacional de Chile. [Internet].[Consultado Diciembre 2011].Disponible en: http://www.bcn.cl/histley/lfs/hdl20282/HL20282.pdf

17. Valecillo $M$, Quevedo A, Lubo Palma A, Dos Santos A, Montiel M, Camejo M, Sánchez M. Síntomas musculo esqueléticos y estrés laboral en el personal de enfermería de un hospital militar. Salud trab. 2009; 17(2): 85-95.

18. Vilela J, Días T, Sanfeliz A. Análisis ergonómico en enfermería instrumentista: Un enfoque descriptivo. INSHT.2003; 24:5-10.

19. Vernanza-Pinzón P, Sierra-Torres C. Dolor musculo - esquelético y su asociación con Factores de Riesgo Ergonómicos, en Trabajadores Administrativos. Rev. Salud pública.2005; 7(3): 317-26.

20. Morata Ramírez M, Ferrer Pérez V. Interacción entre estrés ocupacional, estrés psicológico y dolor lumbar: un estudio en profesionales sanitarios de traumatología y cuidado intensivos. Mapfre Medicina, 2004; 15:199-211.

21. Peña J.L, Solano A.M. Factores relacionados con la aparición de lumbalgia en las enfermeras. Rev. Medica Sanitas. 2009; 12(4)26-32.

22. Morán Ramírez C. Riesgos laborales del profesional de enfermería en los quirófanos del hospital Sergio E. Bernales - Collique, Febrero-agosto 2009. Rev Per Obst Enf.2009; 5 (2): 104-9. 